\title{
Sensitivity and Specificity of Cystatin C in Detecting Early Renal Impairment in Hypertensive Pregnancies
}

\author{
Fauziah Jummaat ${ }^{1}$, Azreen Syazril Adnan ${ }^{2}$, Nor Aliza Abd Ghaffar ${ }^{1}$, Julia Omar ${ }^{3}$, \\ Syed Hatim Noor ${ }^{4}$, Nurul Jannah Ambak ${ }^{4}$ and Amer Hayat Khan ${ }^{5}$ \\ ${ }^{1}$ Department of Obstetrics \& Gynecology, ${ }^{2}$ CKD Resource Centre, Hospital Universiti Sains Malaysia, ${ }^{3}$ Department of \\ Chemical Pathology, School of Medical Sciences, Universiti Sains Malaysia, Kubang Kerian, 16150, Kelantan, Malaysia, ${ }^{4}$ Unit \\ of Biostatistics and Research Methodology, School of Medical Sciences, Universiti Sains Malaysia, Kubang Kerian, 16150, \\ Kelantan, ${ }^{5}$ Department of Clinical Pharmacy, School of Pharmaceutical Sciences, Universiti Sains Malaysia, 11800 Penang, \\ Malaysia
}

*For correspondence: Email: drfauziahjummaat@gmail.com; Tel: 0060-12-9480982

\begin{abstract}
Purpose: To determine the cutoff point of cystatin $C$ for the detection of renal impairment in hypertensive pregnancies.

Methods: A cross-sectional study was conducted in an antenatal clinic and ward at Hospital Universiti Sains Malaysia, Kelantan, Malaysia from January 2009 until January 2010. Sixty four pregnant patients beginning at 2 nd trimester, aged of 16 to 55 years and hypertensive, including gestational hypertension, chronic hypertension with superimposed preeclampsia, preeclampsia and unclassified hypertension, were included in the study. Consenting patients were required to provide $5 \mathrm{ml}$ of blood and 24-h urine. Serum and reagent, $N$ Latex cystatin $C$, were equilibrated at room temperature and measured by particle-enhanced nephelometric immunoassay (PENIA) using a BN II Dade Behring Nephelometer System

Results: The mean age of the patients was $37.06 \pm 4.32$ (range: 24 to 46 years). A majority (64.1\%) of the patients were in the second trimester of pregnancy and delivered in the gestational period of $38-40$ weeks (54.7\%). The number of patients in chronic kidney disease (CKD) stages I, II, III, IV and V were $25(39.1 \%), 18(28.1 \%), 18(28.1 \%), 2(3.1 \%)$ and 1 (1.6 \%), respectively. The mean systolic blood pressure was $149.59 \pm 18.79 \mathrm{~mm} \mathrm{Hg}$, and diastolic blood pressure $91.53 \pm 10.33 \mathrm{~mm} \mathrm{Hg}$. The cutoff point in detecting renal impairment using cystatin $C$ was $>0.74$ with $84.6 \%$ sensitivity and $86.7 \%$ specificity for second trimester and $>0.81$ with sensitivity of $76.9 \%$ and specificity of $60.0 \%$ in detecting renal impairment for third trimester.

Conclusion: The cutoff point in detecting renal impairment for second trimester is better than for third trimester since it maximizes the value of sensitivity and specificity.
\end{abstract}

Keywords: Cystatin C, Sensitivity, Specificity, Renal impairment, Hypertension; Pregnancy

Tropical Journal of Pharmaceutical Research is indexed by Science Citation Index (SciSearch), Scopus, International Pharmaceutical Abstract, Chemical Abstracts, Embase, Index Copernicus, EBSCO, African Index Medicus, JournalSeek, Journal Citation Reports/Science Edition, Directory of Open Access Journals (DOAJ), African Journal Online, Bioline International, Open-J-Gate and Pharmacy Abstracts

\section{INTRODUCTION}

Literature shows that patients with hypertension had a significant higher risk of developing chronic kidney disease compared with normotensive patient [1]. Kidney is an important organ in maintaining physiology of pregnancy. Pregnancy- related acute renal failure predisposes the pregnancy to multiple medical risks, thus increased morbidity and mortality [2]. Preeclampsia and uncontrolled hypertension are recognized factors causing renal impairment. In third world countries pregnancy causes $25 \%$ of acute kidney injury and maternal mortality rate 
ranges from 9 - $55 \%$ [3]. Preeclampsia increases maternal morbidity and mortality [4]. Renal impairment is associated with hypertensive pregnancies [5]. Early detection of renal impairment in hypertensive pregnancies enables immediate referral and multidisciplinary approach. Consequently, further deterioration of kidney function is preventable. A diagnostic tool with high sensitivity and acceptable specificity is important for renal function assessment [6].

Preeclampsia and eclampsia constitutes $75 \%$ of all causes of acute kidney injury in pregnancy, while sepsis $(11 \%)$ is followed by hemorrhage (7.2 \%) [7]. Furthermore, fetal death is reported as $5.5 \%$ with maternal death at $9.1 \%$ [4]. Another study conducted in India, discovered maternal mortality due to pregnancy related acute renal failure to be $24.3 \%$ [7]. Perinatal mortality rate is significantly higher with glomerular filtration rate (GFR) of $70 \mathrm{ml} / \mathrm{min}$ [4]. A consistent observation showing that serum creatinine $>2.5 \mathrm{mg} / \mathrm{dl}(220 \mu \mathrm{mol} / \mathrm{l})$ is associated with more preterm deliveries and lower birth weight than women with a lower serum creatinine. A majority (73 \%) of such women were delivered before 37 weeks and $57 \%$ had intra-uterine growth retardation [8] . It is highly important to diagnose and assess renal function in pregnant patients rapidly and at early stage.

The study was designed to determine the cutoff point of Cystatin $\mathrm{C}$ for the detection of renal impairment in hypertensive pregnancy in second and third trimesters.

\section{EXPERIMENTAL}

\section{Patients and samples}

A cross sectional study was conducted in Hospital USM from January 2009 until January 2010. All pregnant patients beginning with $2^{\text {nd }}$ trimester and hypertensive including gestational hypertension, chronic hypertension, and chronic hypertension with superimposed preeclampsia, preeclampsia and unclassified hypertension were invited to participate, as long as the patients fit the inclusion and exclusion criteria. Patients were excluded if they were hyper/hypothyroidism, smoker, suffering from any forms of malignancy or receiving corticosteroids treatment (which can cause changes in serum cystatin $\mathrm{C}$ ). Patients were fully informed regarding their involvement and the needs to collect $5 \mathrm{ml}$ of blood sample exclusive of other routine blood investigation. Recruitment was done on patients that meet the inclusion and exclusion criteria and consented to participate. Blood sample was taken in $2^{\text {nd }}$ and $3^{\text {rd }}$ trimester and 24 hour urine was collected once in the study. Study was approved by the
Research Ethics Committee (Human) of the Institutional Review Board of the University Sains Malaysia (USMKK/PPP/JEPeM 205.4[2.5]) and funded by university short-term grant.

\section{Laboratory analysis}

Blood samples were taken to the Department of Chemical Pathology Laboratory, School Of Medical Sciences for analysis where they were run in an Eppendorf Centrifuge 5810 Machine to obtain serum and supernatant. The sera were stored at $-17^{\circ} \mathrm{C}$ until they were used. Serum and reagent $\mathrm{N}$ Latex cystatin $\mathrm{C}$ were equilibrated in room temperature and measured with a particleenhanced nephelometric immunoassay (PENIA) using a BN II Dade Behring Nephelometer System. Each sample was diluted to 1:100 with a diluent (N Latex Cystatin $\mathrm{C}$ Kit). Serum and urine creatinine were analyzed by a kinetic colorimetric assay on a Hitachi 912 Analyzer.

\section{Statistical analysis}

All statistical analyses were performed using SPSS 17.0 (SPSS Inc.). Descriptive analysis was performed to categories mean age of patients, patient trimester and stage patients according to CKD. Sensitivity and specificity for cystatin C was assessed by the Receiver Operating Characteristic (ROC) curves.

\section{Ethical approval}

The current study was approved by USM ethical committee USMKK/PPP/JEPeM (205.4\{2.5\})

\section{RESULTS}

The mean age of hypertensive patients was $37.06 \pm 4.32$ with a range of 24 to 46 years. A majority of patients were in the second trimester of pregnancy $(64.1 \%)$ and delivery in the gestational range of 38 - 40 weeks (54.7\%). Number of patients in CKD stages I, II, III, IV and V were 25 (39.1\%), 18 (28.1\%), 18 (28.1\%), 2 $(3.1 \%)$ and 1 (1.6\%), respectively. The mean systolic blood pressure was $149.59 \pm 18.79 \mathrm{~mm}$ $\mathrm{Hg}$ and diastolic blood pressure $91.53 \pm 10.33$ $\mathrm{mm} \mathrm{Hg}$.

CKD stage was defined based on creatinine clearance value. For second trimester, ROC curve identified the level of cystatin $C$ between 0.574 and $0.898(p=0.012)$ (Figure 1) and area under curve was 0.736 . Therefore, the cutoff point in detecting renal impairment using Cystatin C was more 0.74 with $84.6 \%$ sensitivity and $86.7 \%$ specificity (Table 1 ). 
Table 1: Cutoff values of serum Cystatin C for each trimester

\begin{tabular}{lccccc}
\hline Trimester & Area under ROC curve & $\boldsymbol{P}$-value & Cutoff point & Se & Sp \\
\hline Second & 0.736 & 0.012 & $\geq 0.74$ & 84.6 & 86.7 \\
Third & 0.838 & 0.006 & $\geq 0.81$ & 76.9 & 60.0 \\
\hline$R O C$
\end{tabular}

$R O C=$ receiver operating characteristics curve; $S e=$ Sensitivity; $S p=$ Specificity

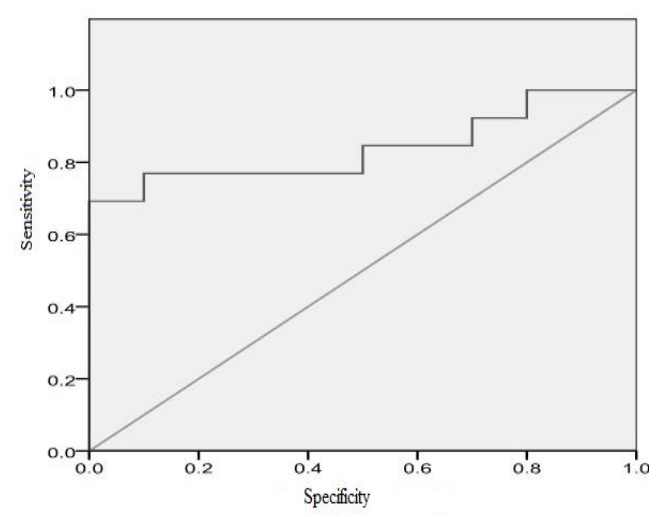

Figure 1: ROC curve for cystatin $C$ in second trimester

The ROC curve drawn in the third trimester revealed cystatin $C$ value of between 0.657 and 1.000 , and represents area under the curve of $0.838(p=0.006)$ (Figure 2).

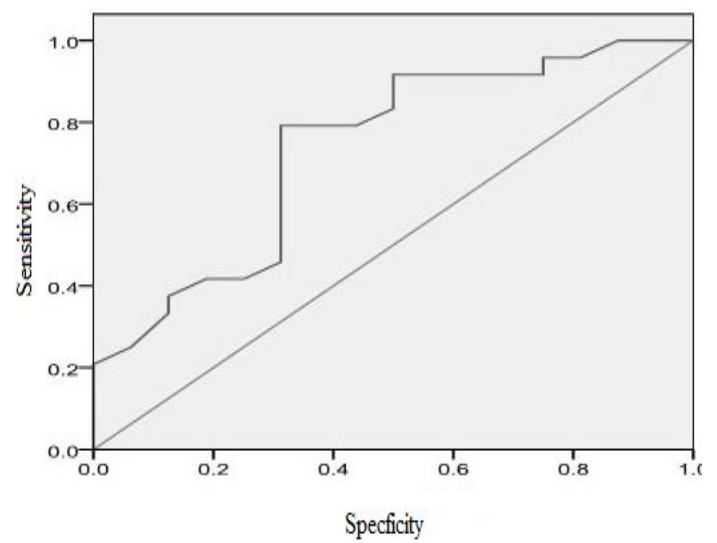

Figure 2: ROC curve for cystatin $\mathrm{c}$ in third trimester

The sensitivity of cystatin C was $76.9 \%$ and while specificity was $60.0 \%$ and cutoff point in detecting renal impairment was $>0.81$ (Table 1). The scattered plots demonstrate the downward trend of creatinine clearance in these patients (Figure 3).

\section{DISCUSSION}

The study shows that cystatin $\mathrm{C}$ has acceptable sensitivity and specificity in detecting renal impairment in hypertensive pregnancies. In view of the variation in cystatin $C$ levels throughout tpregnancy, two different upper limits of cystatin $\mathrm{C}$ were used as reference values. Therefore, the sensitivity and specificity of cystatin $C$ differs as in the second and third trimesters. In second trimester the upper limit is 0.65 while in the third trimester, the upper limit is 0.82. Creatinine clearance of below $90 \mathrm{ml} / \mathrm{min}$ is taken as renal impairment [9]

Another study by Strevens et al carried out in Europe in the pre-eclampsia population determined the reference value of cystatin $\mathrm{C}$ to detect renal failure in this group of patients [10]. Strevens et al found that the sensitivity and specificity of cystatin $\mathrm{C}$ in pre-eclampsia population was 91.1 and $86.6 \%$, respectively.

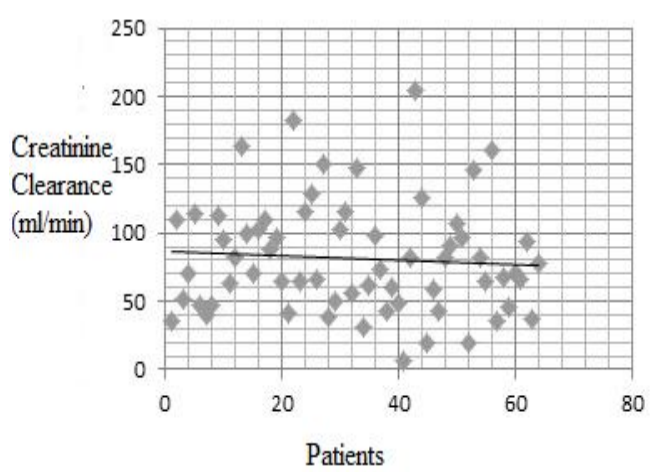

Figure 3: Creatinin clearance of Cystatin $C$ in patients

Discrepancies in outcomes between Strevens's findings and those of the present study are mainly due to the inclusion of subjects from second and third trimesters and use of cutoff point of Cystatin $\mathrm{C}$ for the analysis of sensitivity and specificity. In Strevens et al's study, 45 preeclamptic patients were included and the patients were in second and third trimesters. In current study 64 patients were included, forty one patients in the second trimester and twenty three in the third trimester. Strevens et al, compared serum cystatin C level and serum creatinine, whereas in our study, comparison was made between cystatin $C$ and 24-h creatinine clearance. Dunlop et al stated that serum creatinine measurement has a disadvantage especially during pregnancy as serum creatinine decreases as a result of increased renal plasma flow and GFR during pregnancy [11].

Another explanation for the different value of sensitivity and specificity obtained in present study compared to other studies was subgroup into two different trimesters. While in the study by Strevens et al., patients were taken collectively and their gestational age or trimesters were not considered. Therefore the value of sensitivity and 
specificity is a generalization throughout the pregnancy. Whereas in current study patient were classified based on their gestational age or trimester. In present study, 41 and 23 patients were included in second and third trimester of pregnancy, respectively. However there was unequal distribution in the number of patients between the two trimesters. There was lower number of patients in the third trimesters. Therefore such imbalance may affect the accuracy of the sensitivity and specificity of Cystatin C. This may explain the lower sensitivity and specificity of Cystatin $\mathrm{C}$ in the third trimester.

The variability of Cystatin $C$ level as described by Babay et al, should be taken into consideration to avoid inaccuracy. In this study, 197 normal pregnancies patients were included and cystatin $C$ were measured. The objective of this study is to look at the mean serum Cystatin $C$ value in normal pregnancies. In this study, mean serum Cystatin $\mathrm{C}$ for all the women was $0.82 \pm 0.18$ mg/l. Serum Cystatin C levels were high $0.89 \pm$ $0.12 \mathrm{mg} / \mathrm{l}$ during the first trimester, decreased significantly to $0.65 \pm 0.14 \mathrm{mg} / \mathrm{l}$ during the second trimester $(p<0.001)$ compared to first trimester, and increased again to $0.82 \pm 0.19$ $\mathrm{mg} / \mathrm{l}$ in the third trimester.

Study was unable to include patients in their first trimester population, mainly due to our hospital setting as the tertiary referral center. Most cases accepted from the peripheries were in their second and third trimesters. The lower sensitivity and specificity value of cystatin $C$ in the third trimester in this study can be explained by the smaller sample size which was twenty three patients in third trimester group and inclusion of one patient of End Stage Renal Failure.

We found the mean birth weight of babies delivered in present study to be $2.73 \pm 0.71 \mathrm{~kg}$, and 1 delivery was an intrauterine death. The birth weight which is acceptable in our population can be explained by fairly acceptable blood pressure control in this study. Majority of the studied subjects only require a single antihypertensive agent to optimize their blood pressure control. Small proportion of the studied subjects requires two agents to control their blood pressure. The combined pair of antihypertensive agents was primarily labetolol with either nifedipine or methyldopa.

This acceptable range of blood pressure control resulted in better outcomes measured by the acceptable birth weight. Single intrauterine death was from a severe pre-eclampsia mother. Out of that, 27 cases underwent Caesarean section. The indications were mainly due to pre- eclampsia, sixteen (59 \%) patients and acute fetal distress, nine (33 \%) patients. A small proportion about $2(7 \%)$ of these patients had to be operated due to their past history of 2 previous scars. However this study is not powered to look at the correlation between the cystatin $\mathrm{C}$ and the outcomes of these pregnancies.

The natural history of kidney deterioration in preexisting renal impairment patients whom are pregnant and hypertensive were also demonstrated in this study. Uncontrolled hypertension predispose to intraglomerular endothelisosis and thus deterioration of glomerular filtration rate. This study however is not meant to review the renal function of these patients post delivery. The pregnancy itself may serve as the potential 'stressors' to the kidney. Cystatin C level increased throughout with the gestational age. This finding also supports the findings of a study carried by Babay et al [12]. It will be helpful for future study to review cystatin C level post-delivery. This observational study if carried out will be able to demonstrate potential reversibility post-delivery. Furthermore, Strevens et al, discovered cystatin C level is proportionately increased throughout pregnancy, which buttresses the findings of the present study.

\section{CONCLUSION}

This method has the advantage of detecting renal impairment in a fairly wide age group presenting with hypertensive pregnancies. A majority of these patients were between ages of 30-33 years old. In our daily clinical practice majority of hypertensive pregnancies came from this particular age group. The potential use of cystatin $\mathrm{C}$ for early detection of renal impairment is valid in this study. However, a drawback is the cost as the laboratory should be equipped with ELISA reader.

\section{REFERENCES}

1. Coresh J, Astor BC, Greene T, Eknoyan G, Levey, AS. Prevalence of chronic kidney disease and decreased kidney function in the adult US population: Third National Health and Nutrition Examination Survey. Am J Kidney Dis, 2003; 41(1): 1-12.

2. Brown, MA, Lindheimer Marshall D, Swiet D, Michael, Assche V, Andre V, Moutquin, Jean-Marie., The classification and diagnosis of the hypertensive disorders of pregnancy: statement from the International Society for the Study of Hypertension in Pregnancy (ISSHP). Hypertension in pregnancy, 2001; 20(1): 9-14.

3. Prakash J, Tripathi K, Malhotra V, Kumar O, Srivastava PK. Acute renal failure in eastern India. Nephrol Dial Transplant, 1995; 10(11): 2009-2012. 
4. Geraldo B. Silva Jr, Amaury F, Monteiro, Rosa M. S , Gerardo J.A, Walter Correia J, José G, Bezerra F, Rafael N. Macedo, Rafael S. A, Elizabeth F. D Acute kidney injury requiring dialysis in obstetric patients: a series of 55 cases in Brazil. Arch Gynecol Obstet, 2009; 279(2): 131-137.

5. Chee YC. Update on management of hypertension in pregnancy--medical aspects. Singapore Med J, 1988; 29(1): 24-27.

6. Hoek FJ, Kemperman FA, Krediet FT. A comparison between cystatin $C$, plasma creatinine and the Cockcroft and Gault formula for the estimation of glomerular filtration rate. Nephrol Dial Transplant, 2003; 18(10): 2024-2031.

7. Misra R, Bhowmik D, Mittal S, Kriplani A, Kumar S, Bhatla N, Dadhwal V, Pandey RM., Pregnancy with chronic kidney disease: outcome in Indian women. J Womens Health (Larchmt), 2003; 12(10): 10191025.

8. Knutzen, V.K. and D.A. Davey, Hypertension in pregnancy and perinatal mortality. S Afr Med J, 1977; 51(19): 675-679.
9. Bertolatus JA, GL. Evaluation of renal function in potential living kidney donors. . Transplantation, 200171 : 256-260.

10. Strevens $H$, Wide-Swensson $D$, Torffvit $O$, Grubb $A$. Serum cystatin $C$ for assessment of glomerular filtration rate in pregnant and non-pregnant women. Indications of altered filtration process in pregnancy. Scand J Clin Lab Invest. 2002; 62: 141-147.

11. Davison JM, Dunlop W: Changes in renal hemodynamics and tubular function induced by normal pregnancy. Semin Nephrol 4: 198, 1984, 198-204.

12. Babay Z ,Al-Wakeel J, Addar M, Mittwalli A, Tarif N, Hammad D, Ali N, Al-Askar A, Choudhary AR., Serum cystatin $C$ in pregnant women: reference values, reliable and superior diagnostic accuracy. Clin Exp Obstet Gynecol, 2005; 32(3): 175-179. 